\title{
Infant neurocognitive development is independent of the use of iodised salt or iodine supplements given during pregnancy
}

\author{
Piedad Santiago ${ }^{1}$, Inés Velasco ${ }^{2}$, Jose Antonio Muela ${ }^{3}$, Baltasar Sánchez ${ }^{4}$, Julia Martínez ${ }^{5}$, \\ Alvaro Rodriguez ${ }^{3}$, María Berrio ${ }^{1}$, Carolina Gutierrez-Repiso ${ }^{6}$, Mónica Carreira ${ }^{7}$, Alberto Moreno ${ }^{1}$, \\ Eduardo García-Fuentes ${ }^{6,8,9 *}$ and Federico Soriguer ${ }^{6,8,9,10}$ \\ ${ }^{1}$ Servicio de Endocrinología y Nutrición, Complejo Hospitalario de Jaén, Jaén, Spain \\ ${ }^{2}$ Servicio de Ginecología y Obstetricia, Hospital de Riotinto, Huelva, Spain \\ ${ }^{3}$ Departamento de Psicología, Universidad de Jaén, Jaén, Spain \\ ${ }^{4}$ Servicio de Análisis Clínicos, Complejo Hospitalario de Jaén, Jaén, Spain \\ ${ }^{5}$ Servicio de Radiología, Complejo Hospitalario de Jaén, Jaén, Spain \\ ${ }^{6}$ Instituto de Investigación Biomédica de Málaga (IBIMA), Málaga, Spain \\ ${ }^{7}$ Facultad de Psicología, Universidad de Málaga, Málaga, Spain \\ ${ }^{8}$ Servicio de Endocrinología y Nutrición, Hospital Regional Universitario Carlos Haya, Plaza del Hospital Civil s/n, \\ 29009 Málaga, Spain \\ ${ }^{9}$ CIBEROBN, Málaga, Spain \\ ${ }^{10}$ CIBERDEM, Málaga, Spain
}

(Submitted 9 August 2012 - Final revision received 4 December 2012 - Accepted 8 December 2012 - First published online 4 February 2013)

\section{Abstract}

The benefits of iodine supplements during pregnancy remain controversial in areas with a mild-to-moderate iodine deficiency. The aim of the present study was to determine the effect of improving iodine intakes, with iodised salt (IS) or iodine supplements, in pregnant Spanish women. A total of 131 pregnant women in their first trimester were randomly assigned to three groups: (1) IS in cooking and at the table, (2) $200 \mu \mathrm{g}$ potassium iodide (KI)/d or (3) $300 \mu \mathrm{g} \mathrm{KI} / \mathrm{d}$. No differences were found in thyroid-stimulating hormone (TSH), free thyroxine (FT4), free triiodothyronine (FT3) or thyroid volume (TV) between the three groups. Regardless of the group in which women were included, those who had been taking IS for at least 1 year before becoming pregnant had a significantly lower TV in the third trimester $(P=0.01)$ and a significantly higher urinary iodine in the first $(173.7$ (SD 81.8) $v .113 .8$ (sD 79.6) $\mu \mathrm{g} / 1, P=0.001)$ and third trimesters (206.3 (SD 91.2) v. 160.4 (sD 87.7) $\mu \mathrm{g} / 1, P=0 \cdot 03$ ). Also, no differences were seen in TSH, FT4 or FT3. Children's neurological development was not significantly associated with the consumption of IS for at least 1 year before becoming pregnant and no differences were found according to the treatment group. In conclusion, in pregnant women with insufficient iodine intake, the intake of IS before becoming pregnant was associated with a better maternal thyroid function. The form of iodide intake was not associated with maternal thyroid function or children's neurological development.

Key words: Iodine: Pregnancy: Thyroid function: Neuropsychomotor development

The relationship between nutritional iodine deficiency during pregnancy and the neurocognitive development of children has been determined in areas of severe iodine deficiency ${ }^{(1)}$, and even in areas that experience just a moderate dietary iodine deficiency ${ }^{(2)}$. An adequate level of maternal thyroid hormones is required during pregnancy to ensure transfer to the fetus of these hormones, which are vital for the optimal neuromotor development of the offspring ${ }^{(3)}$.
Studies on the effect of isolated hypothyroxinaemia on the neurocognitive development of children have produced discordant results. While some studies have shown that maternal hypothyroxinaemia can result in delayed mental development in children ${ }^{(4)}$, other studies have found that isolated hypothyroxinaemia during the second trimester is not associated with significantly lower Bayley Scale Infant Development (BSID)-III scores at the age of 2 years,

Abbreviations: BSID, Bayley Scale Infant Development; FT3, free triiodothyronine; FT4, free thyroxine; IS, iodised salt; MDI, Mental Development Index; MDS, Mental Development Scale; PDI, Psychomotor Development Index; PDS, Psychomotor Development Scale; TDI, Total Development Index; TSH, thyroid-stimulating hormone; TV, thyroid volume.

*Corresponding author: E. G. Fuentes, fax +34 95228670, email edugf1@gmail.com 
when compared with the scores for offspring of matched euthyroxinaemic women ${ }^{(5)}$.

Recent years have seen a certain international agreement on the systematic recommendation for iodised salt (IS) as a means to iodisation at the population level. In particularly vulnerable groups, such as pregnant women, the use of pharmacological iodine supplements has been recommended in areas that, historically, had experienced a nutritional iodine deficiency and where IS is consumed in fewer than $90 \%$ of homes ${ }^{(6)}$. Many reasons have been given for these recommendations, basically because, on the one hand, it is not easy to identify the individual risk for iodine deficiency and, on the other hand, the recommended iodine doses in prevention programmes are significantly lower than the potentially harmful doses $^{(7)}$. Recently, a median urinary iodine concentration $<150 \mu \mathrm{g} / 1$ has been proposed to classify pregnant women with insufficient iodine intake ${ }^{(8,9)}$

Despite these recommendations, the benefits of iodine supplements during pregnancy remain controversial. Rebagliato et $a l .{ }^{(10)}$ showed that iodine supplement intake in the first half of pregnancy may lead to maternal thyroid dysfunction in iodine-sufficient or mildly iodine-deficient populations. Controlled trials of iodine supplementation in mildly iodinedeficient pregnant women suggest beneficial effects on maternal and newborn serum thyroglobulin and thyroid volume (TV), but no effects on maternal and newborn total or free thyroid hormone concentrations ${ }^{(8)}$. There are no long-term data on the effect of iodine supplementation on birth outcomes or infant development ${ }^{(8)}$. In a previous study, we have shown that children whose mothers with insufficient iodine intake receiving $300 \mu \mathrm{g}$ iodine daily from the first trimester had a more favourable psychomotor evaluation than those whose mothers were not treated. Also, in this population with insufficient iodine intake, the prescription of iodine supplements, at least in the dose used, is able to reduce the usual decline in free thyroxine (FT4) during pregnancy $^{(2)}$. Indeed, thyroid dysfunction during late gestation has been associated with excessive iodine intake in pregnant women $^{(11)}$. However, the benefit of iodine supplementation in severe iodine deficiency is well established ${ }^{(8,12)}$.

Therefore, the aim of the present study was to determine the effect of three different forms of iodine intake on maternal and neonatal thyroid function, the TV during pregnancy and the neuropsychomotor development of the children of these mothers in a population of pregnant women with insufficient iodine intake.

\section{Materials and methods}

\section{Study subjects}

The study comprised 131 women selected before week 10 of pregnancy from a healthy pregnancy programme at primary care centres in Jaen (Spain). None of the women had taken iodine supplements before the start of the present study. A sequence of random numbers was generated with appropriate software for this purpose. Pregnant women were assigned to one of the following three intervention groups after meeting the inclusion criteria: (1) the IS group ( $n$ 38); (2) the group receiving supplementation with $200 \mu \mathrm{g}$ iodide/d in the form of potassium iodide (KI) ( $n$ 55); (3) the group receiving supplementation with $300 \mu \mathrm{g} \mathrm{KI} / \mathrm{d}$ ( $n$ 38). Women in the IS group received recommendations to replace common cooking and table salt with IS. This group took no pharmacological iodine supplements during pregnancy. In all the cases, supplementation was started before week 10 of gestation. Women in the 200 and $300 \mu \mathrm{g}$ groups also received the recommendation to consume IS if they were not already doing so. Folic acid was prescribed at the same doses to all pregnant women $(0.4 \mathrm{mg} / \mathrm{d})$, regardless of $\mathrm{KI}$, to prevent neural tube defects. Based on previous results from our group ${ }^{(2,13)}$, sample size was calculated from the urinary iodine, FT4, Psychomotor Development Index (PDI) and TV of the study population. For an $\alpha=0.05$ and $\beta=0.80$, the number of cases required to detect a difference in urinary iodine $(15 \mu \mathrm{g} / \mathrm{l})$ and FT4 $(0.233 \mathrm{pmol} / \mathrm{l})$ between the groups is ninety ( $n 30$ per group). To detect a difference in PDI (8.0) between the groups, the number of cases required is seventy-eight ( $n 26$ per group). To detect a difference in TV $\left(2 \mathrm{~cm}^{3}\right)$ between the groups, the number of cases required is sixty ( $n 20$ per group). The total number of recruited cases was 168 women ( $n 56$ per group). However, some women did not complete the study throughout pregnancy for different reasons. This group of pregnant women had at baseline a similar age, weight, urinary iodine, thyroid-stimulating hormone (TSH), FT4, free triiodothyronine (FT3), thyroglobulin and TV compared with pregnant women who completed the study (data not shown). The present study was approved by the Ethics and Research Committee of Jaen Hospital, and written informed consent was obtained from all participants. The study complies with the principles laid down in the Declaration of Helsinki.

All the women provided a blood sample to measure their thyroid function and a first morning urine sample for the measurement of urinary iodine. They also underwent a thyroid echogram to measure TV during the first trimester (before week 10 and before assignment to their group), as well as during the second trimester (24-28 weeks) and the third trimester (week 36). Of the 131 women, forty-four (IS group, $n$ 13; $200 \mu \mathrm{g}$ group, $n$ 20; $300 \mu \mathrm{g}$ group, $n$ 11) provided a sample of breast milk during the first $48 \mathrm{~h}$ after delivery in order to measure iodine in the milk. In addition, seventynine underwent repeat laboratory tests between 3 and 6 months postpartum to study their thyroid function (IS group, $n$ 25; $200 \mu \mathrm{g}$ group, $n$ 26; $300 \mu \mathrm{g}$ group, $n$ 28). Obstetric information was also gathered for all the women by a chart review, and the women were asked directly in primary care centres and by telephone about the type of salt they usually consumed. The compliance of IS intake or KI was also asked by telephone. Those women with anti-thyroid antibodies and TSH concentrations $>5 \mu \mathrm{IU} / \mathrm{ml}(>5 \mathrm{mIU} / \mathrm{l})$ were excluded from the study analysis.

Information collected about the newborns included anthropometric data and Apgar score, as recorded in the chart of the mother. Cord blood TSH was obtained from the congenital hypothyroidism screening programme. In addition, forty-one 
children (IS group, $n$ 11; $200 \mu \mathrm{g}$ group, $n$ 15; $300 \mu \mathrm{g}$ group, $n$ 15) underwent thyroid echography during the first week after birth. Finally, between the ages of 6 and 18 months, 102 children underwent psychometric assessment (IS group, $n$ 25; $200 \mu$ g group, $n$ 47; $300 \mu$ g group, $n$ 30).

\section{Laboratory procedures}

Thyroid hormones and thyroglobulin were measured by a chemoluminescent immunoassay for the quantitative determination of the concentrations of TSH, FT4, FT3 and thyroglobulin in a Beckman Access ${ }^{\circledR} 2$ Immunoassay System (Beckman Coulter, Inc.). Reference ranges (percentile 3-percentile 97) for TSH are as follows: first trimester, 0.23-4.18 $\mu \mathrm{UI} / \mathrm{ml}$; second trimester, $0.36-3.89 \mu \mathrm{UI} / \mathrm{ml}$; third trimester, $2 \cdot 01-4 \cdot 30 \mu \mathrm{UI} / \mathrm{ml}^{(14)}$. Reference ranges for FT4 are as follows: first trimester, $6 \cdot 0-10.6 \mathrm{ng} / \mathrm{l}$; second trimester, $4.3-8.5 \mathrm{ng} / \mathrm{l}$; third trimester, $4 \cdot 0-8 \cdot 2 \mathrm{ng} / \mathrm{l}^{(14)}$. Reference ranges for FT3 are as follows: first trimester, $2.33-3.84 \mathrm{pg} / \mathrm{ml}$; second trimester, $2 \cdot 04-3.51 \mathrm{pg} / \mathrm{ml}$; third trimester, $1.99-3.46 \mathrm{pg} / \mathrm{ml}^{(14)}$. Reference ranges for thyroglobulin are as follows: first trimester, $1 \cdot 18-61.51 \mathrm{ng} / \mathrm{ml}$; second trimester, $1.92-37.98 \mathrm{ng} / \mathrm{ml}$; third trimester, $1.69-59 \cdot 87 \mathrm{ng} / \mathrm{ml}^{(14)}$. Antiperoxidase antibodies (thyroid peroxidase) were measured by a radioimmunometric assay (Biocode S.A.). Iodine in maternal milk and first morning urine was measured by the method of Benotti \& Benotti $^{(2,15,16)}$. TV was measured by real-time ultrasound using a $7.4 \mathrm{MHz}$ linear transducer ${ }^{(17)}$. TV was measured using the following calculation: $\mathrm{TV}(\mathrm{ml})=\mathrm{TV}$ of the right lobe $(\mathrm{ml})+\mathrm{TV}$ of the left lobe $(\mathrm{ml})$. The volume of each lobe was measured using the following calculation: lobe volume $(\mathrm{ml})=$ long axis $(\mathrm{cm}) \times$ short axis $(\mathrm{cm}) \times$ thickness $(\mathrm{cm}) \times 0.479$.

\section{Evaluation of children's neurological development}

Children were assessed at a single session in the presence of their mother or father, between the ages of 6 and 18 months. Psychological assessment was done by an independent investigator blind to the type of the study design. The assessment method comprised two procedures: (1) a structured interview with the mother or the father to collect sociodemographic data; (2) BSID-III ${ }^{(2)}$. The Bayley Scales are a method to measure the specific development of children aged 2-30 months; the method consists of three scales: Mental Development Scale (MDS); Psychomotor Development Scale (PDS); Behavioral Development Scale ${ }^{(2)}$. Children's scores on the test give direct scores. The scores on the MDS and the PDS enable direct comparison between the subjects, though they have the inconvenience of being affected by the age at the time of the assessment. Accordingly, these quantitative scales are transformed into indices: Mental Development Index (MDI) and PDI, respectively. The sum of these two indices is the Total Development Index (TDI). The resulting indices (MDI, PDI and TDI) are typical normalised scores (they indicate the number of standard deviations by which a score is above or below the mean of a normal distribution), and form what is called an interval scale. Measurements on this scale reflect delay, precocity or similarity in relation to the functioning level of persons of the same chronological age.

\section{Statistical analysis}

Data are presented as means and standard deviations. Continuous variables were adjusted to the normality of distribution with the Shapiro test. Hypothesis contrast between two samples was done with Student's $t$ test, Wilcoxon test or Mann-Whitney test. Classification variables of more than two levels were examined with ANOVA and Kruskal-Wallis tests, depending on the adjustment to the normality of distribution. The dependence between variables was evaluated with Pearson's or Spearman's correlation coefficients, depending on the adjustment to normality. An ANOVA for repeated measures was performed in those cases in which we analysed simultaneously three trimesters. In all cases, the level of rejection was $\alpha=0.05$ for two tails. Statistical analysis was done with SPSS (version 11.5 for Windows; SPSS, Inc.).

\section{Results}

The variables related to the mother and the newborn for the three intervention groups are summarised in Table 1 . The age and the number of weeks' gestation of the mothers did not differ depending on the group to which the mothers were assigned.

The mean urinary iodine level of all the women in the first trimester (before being assigned to their groups) was 131.4 (SD 81.9) $\mu \mathrm{g} / \mathrm{l}$ (median $109 \mu \mathrm{g} / \mathrm{l}$ ); no significant differences were found between the groups (Table 1). Urinary iodine levels were significantly higher in the second and third trimesters of pregnancy, and in the group treated with 200 and $300 \mu \mathrm{g}$ KI (Table 1). In an ANOVA model for repeated measures, urinary iodine was significantly different between the trimesters $(P=0.001)$ and between the groups $(P=0.01)$, without a significant trimester $\times$ treatment group interaction $(P=0 \cdot 15)$.

TSH was slightly increased in the third trimester, without differences between the groups (Table 1). In an ANOVA model for repeated measures, TSH was almost significantly increased depending on the trimester $(P=0 \cdot 06)$, and without differences depending on the treatment group $(P=0.50)$. Also, no significant trimester $\times$ group interaction $(P=0.59)$ was found. FT4 decreased significantly during pregnancy $(P<0 \cdot 0001)$, without differences depending on the treatment group $(P=0 \cdot 16)$ and with no significant trimester $\times$ group interaction $(P=0.64)$ (Table 1$)$. FT3 decreased significantly during pregnancy $(P<0 \cdot 0001)$, without differences depending on the treatment group $(P=0.06)$ and with no significant trimester $\times$ group interaction $(P=0.94)$ (Table 1$)$. Thyroglobulin levels did not differ significantly depending on the treatment group $(P=0 \cdot 013)$ or the trimester $(P=0 \cdot 21)$ (Table 1$)$.

During pregnancy, the increase in TSH ((third-trimester TSH - first-trimester TSH)/first-trimester TSH; $P=0$-49), the reduction in FT4 ((first-trimester FT4 - third-trimester FT4)/ first-trimester FT4; $P=0.74)$, and the reduction in FT3 ((firsttrimester FT3 - third-trimester FT3)/first-trimester FT3; $P=0.55)$ did not differ significantly between any of the three 
Table 1. Maternal and infant study variables according to treatment group (Mean values and standard deviations)

\begin{tabular}{|c|c|c|c|c|c|c|c|}
\hline & \multicolumn{2}{|c|}{ lodised salt } & \multicolumn{2}{|c|}{$200 \mu \mathrm{g} \mathrm{KI}$} & \multicolumn{2}{|c|}{$300 \mu \mathrm{g} \mathrm{KI}$} & \multirow[b]{2}{*}{$P$} \\
\hline & Mean & SD & Mean & SD & Mean & SD & \\
\hline$n$ & \multicolumn{2}{|c|}{38} & \multicolumn{2}{|c|}{55} & \multicolumn{2}{|c|}{38} & \\
\hline Age of the mother (years) & $31 \cdot 0$ & $5 \cdot 7$ & $31 \cdot 3$ & 4.4 & $30 \cdot 3$ & 4.4 & 0.854 \\
\hline Length of pregnancy (weeks) & $39 \cdot 28$ & 1.21 & 39.07 & 1.85 & 38.89 & $2 \cdot 61$ & 0.587 \\
\hline \multicolumn{8}{|l|}{ Urinary iodine $(\mu \mathrm{g} / \mathrm{l})$} \\
\hline First trimester & $145 \cdot 1$ & 89.7 & $116 \cdot 5$ & 71.4 & $136 \cdot 9$ & 82.5 & $0.001^{*}$ \\
\hline Second trimester & $130 \cdot 2$ & $64 \cdot 8$ & $177 \cdot 1$ & $82 \cdot 3$ & $222 \cdot 0$ & $85 \cdot 5$ & $0.01 \dagger$ \\
\hline Third trimester & $144 \cdot 2$ & $85 \cdot 4$ & $166 \cdot 1$ & 74.6 & $212 \cdot 4$ & 114.6 & $0.15 \ddagger$ \\
\hline \multicolumn{8}{|l|}{$\mathrm{TSH}(\mu \mathrm{lU} / \mathrm{ml})$} \\
\hline First trimester & 1.61 & 1.01 & 1.76 & 1.29 & 1.75 & 0.91 & $0.06^{\star}$ \\
\hline Second trimester & 1.74 & 0.64 & 1.79 & 1.05 & 2.03 & 0.75 & $0.50 \dagger$ \\
\hline Third trimester & 2.09 & 0.82 & $2 \cdot 11$ & $1 \cdot 30$ & $2 \cdot 51$ & 1.07 & $0.59 \ddagger$ \\
\hline \multicolumn{8}{|l|}{ FT4 (ng/l) } \\
\hline First trimester & $8 \cdot 3$ & 1.3 & $7 \cdot 7$ & $1 \cdot 1$ & $8 \cdot 1$ & 1.7 & $<0.0001^{*}$ \\
\hline Second trimester & $6 \cdot 4$ & $1 \cdot 0$ & 5.9 & 0.9 & $6 \cdot 2$ & 0.9 & $0.16 \dagger$ \\
\hline Third trimester & $6 \cdot 0$ & 1.1 & $5 \cdot 6$ & 1.2 & $6 \cdot 1$ & 1.0 & $0.64 \ddagger$ \\
\hline \multicolumn{8}{|l|}{ FT3 (pg/ml) } \\
\hline First trimester & 3.09 & 0.53 & 2.99 & 0.36 & $3 \cdot 12$ & 0.41 & $<0.0001^{*}$ \\
\hline Second trimester & $2 \cdot 67$ & 0.37 & $2 \cdot 62$ & 0.32 & $2 \cdot 83$ & 0.33 & $0.06 \dagger$ \\
\hline Third trimester & 2.68 & 0.37 & $2 \cdot 72$ & 0.32 & $2 \cdot 76$ & 0.32 & $0.94 \ddagger$ \\
\hline \multicolumn{8}{|l|}{ Thyroglobulin (ng/ml) } \\
\hline First trimester & $21 \cdot 3$ & $42 \cdot 7$ & $25 \cdot 4$ & 71.5 & $13 \cdot 0$ & $9 \cdot 1$ & $0.21^{*}$ \\
\hline Second trimester & $22 \cdot 7$ & 39.2 & $21 \cdot 6$ & $62 \cdot 2$ & 8.4 & $6 \cdot 2$ & $0.13 \dagger$ \\
\hline Third trimester & $22 \cdot 1$ & 37.5 & $24 \cdot 8$ & $75 \cdot 8$ & 13.9 & $14 \cdot 8$ & $0.23 \ddagger$ \\
\hline \multicolumn{8}{|l|}{ Thyroid volume $\left(\mathrm{cm}^{3}\right)$} \\
\hline First trimester & 9.47 & 3.02 & 8.42 & $2 \cdot 77$ & $8 \cdot 86$ & 3.89 & $0.004^{*}$ \\
\hline Second trimester & 8.94 & 2.64 & $9 \cdot 70$ & $3 \cdot 18$ & 8.02 & 3.09 & $0.72 \dagger$ \\
\hline Third trimester & $9 \cdot 72$ & 1.97 & $10 \cdot 79$ & 3.23 & $9 \cdot 50$ & $2 \cdot 76$ & $0.83 \ddagger$ \\
\hline Newborn birth weight (kg) & 3.36 & 0.42 & $3 \cdot 17$ & 0.51 & $3 \cdot 16$ & 0.79 & 0.648 \\
\hline Apgar test & $8 \cdot 71$ & 0.95 & 8.64 & 0.85 & 8.63 & 0.91 & 0.468 \\
\hline Cord blood TSH (mU/ml) & 2.98 & 1.81 & 2.49 & 1.28 & $3 \cdot 22$ & 1.90 & 0.359 \\
\hline Newborn thyroid volume $\left(\mathrm{cm}^{3}\right) \S$ & 0.49 & 0.12 & 0.42 & 0.15 & 0.42 & 0.15 & 0.527 \\
\hline lodine in breast milk $(\mu \mathrm{g} / \mathrm{l}) \|$ & $140 \cdot 8$ & $89 \cdot 6$ & $235 \cdot 3$ & $117 \cdot 2$ & $216 \cdot 2$ & $153 \cdot 9$ & 0.05 \\
\hline Postpartum TSH ( $\mu \mathrm{IU} / \mathrm{ml})$ & 1.44 & 0.83 & 1.59 & 0.73 & 1.76 & 0.70 & 0.215 \\
\hline Postpartum FT4 (ng/l) & $7 \cdot 6$ & 1.7 & $7 \cdot 6$ & 1.4 & $7 \cdot 4$ & 1.6 & 0.845 \\
\hline Postpartum FT3 (pg/ml) & 3.03 & 0.38 & 3.07 & 0.37 & 3.09 & 0.42 & 0.839 \\
\hline Postpartum thyroglobulin (ng/ml) & $16 \cdot 7$ & $31 \cdot 2$ & 14.9 & $10 \cdot 6$ & $18 \cdot 1$ & $29 \cdot 2$ & 0.458 \\
\hline
\end{tabular}

treatment groups (data not shown). In all the cases, the inclusion of urinary iodine levels in any of the three trimesters as a covariable in the different repeated-measures ANOVA models did not improve the explanation for the variance of the changes in TSH, FT4 and FT3 during pregnancy (data not shown).

The TV of the mothers increased slightly but significantly during pregnancy $(P=0.004)$, independently of the treatment group $(P=0 \cdot 72)$, without a significant trimester $\times$ group interaction $(P=0.83)$ (Table 1$)$. TV in the third trimester correlated negatively with urinary iodine levels in the third trimester $(r$ $-0 \cdot 28, P=0.05$, adjusted for FT 4 and the KI dose; Fig. 1(a)), and with TSH in the third trimester $(r-0.30, P=0.03$, adjusted for FT4 and the KI dose; Fig. 1(b)).

The TSH levels of the mothers in the puerperium correlated significantly with the TSH levels in the first $(r 0.30, P=0.007)$, second $(r 0 \cdot 44, P<0 \cdot 001)$ and third $(r 045, P<0 \cdot 0001)$ trimesters.

\section{Consumption of iodised salt before becoming pregnant}

Almost one-third (31.9\%) of the women had been taking IS for at least 1 year before becoming pregnant, and the others started taking it on entering the study. The assignment to the different groups of the women who had been taking IS for at least 1 year before becoming pregnant was 35.6, $19 \cdot 6$ and $40.7 \%$, respectively. The difference was not significant $\left(\chi^{2}=4 \cdot 32, P=0 \cdot 12\right)$.

The women who had been taking IS for at least 1 year had higher urinary iodine levels in the first trimester (173.7 (SD 81.8) $v .113 .8$ (SD 79.6) $\mu \mathrm{g} / 1, P=0.001$, adjusted for the variable treatment group). This difference remained in the third trimester (206.3 (SD 91.2) v. 160.4 (SD 87.7) $\mu \mathrm{g} / 1, P=0.03$, adjusted for the variable treatment group).

No differences were seen in TSH, FT4, FT3 or thyroglobulin throughout the three trimesters according to whether the 

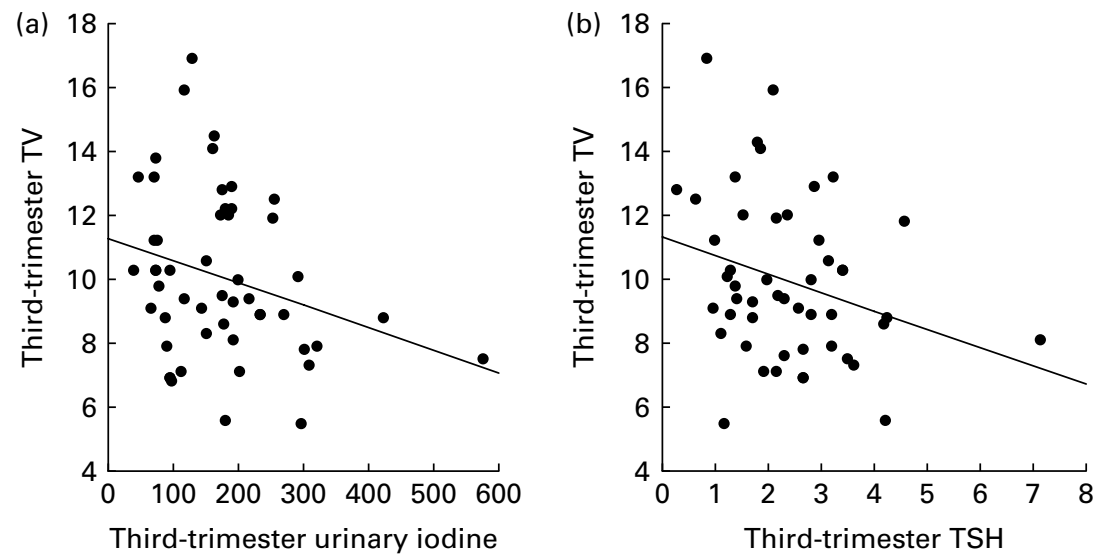

Fig. 1. Correlation between thyroid volume (TV) $\left(\mathrm{cm}^{3}\right)$ in the third trimester and (a) the third-trimester urinary iodine $(\mu \mathrm{g} / \mathrm{l})(r-0.28, P=0.05$, adjusted for the potassium iodide (KI) dose and free thyroxine (FT4)) and (b) the third-trimester thyroid-stimulating hormone (TSH) $(\mu \mathrm{IU} / \mathrm{ml})(r-0.30, P=0.03$, adjusted for the $\mathrm{KI}$ dose and FT4).

women had or had not consumed IS before becoming pregnant (data not shown).

TV in the third trimester was significantly lower in the women who had been taking IS before becoming pregnant ( $P=0 \cdot 01$, adjusted for the variable treatment group; Fig. 2).

\section{Infants}

Table 1 shows that the weight and the TV of the infants, as well as the results of the Apgar test and cord blood TSH levels did not differ significantly according to treatment group. Iodine levels in breast milk were significantly higher in the mothers treated with $\mathrm{KI}(P=0 \cdot 05$; Table 1$)$, correlating significantly with urinary iodine levels in the third trimester ( $r$ 0.34, $P=0.04$ ). Cord blood TSH levels correlated significantly with urinary iodine levels in the third trimester $(r 0 \cdot 40$, $P<0.0001)$, adjusted for gestational age $(P=0.53)$, maternal TSH in the third trimester $(P=0.90)$ and treatment group $(P=0.59)$ (Fig. 3(a)). The TV of the infants correlated negatively and significantly with breast milk iodine concentration ( $r-0 \cdot 43, P=0 \cdot 04$, adjusted for gestational age; Fig. 3(b)).

\section{Psychometric study}

A total of 111 infants underwent the BSID-III test at a mean age of 12.8 (SD 4.8) months, without significant differences between the groups. The age at which this psychometric test was performed correlated significantly with the MDS ( $r 0.93$, $P<0.001)$ and the PDS $(r 0.90, P<0.0001)$, but not with the MDI $(r 0 \cdot 17, P=0 \cdot 10)$, PDI $(r-0 \cdot 01, P=0 \cdot 80)$ or TDI $(r 0.07$, $P=0 \cdot 45$ ), indicating that the indices of development (MDI, PDI and TDI) are independent of the age at which the test is given.

A bivariate analysis showed that the MDS $(P=0.001)$ and the PDS ( $P=0.0001)$, but not the MDI, PDI or TDI, differed significantly according to the treatment group. After adjusting the ANOVA model for gestational age at birth and for the age of the infant at the time the psychometric test was given (Table 2), no significant differences were found in the MDS,
PDS, MDI, PDI or TDI according to the treatment group $(P>0.05$ in all cases).

The variable consumption of IS for at least 1 year before becoming pregnant was neither significantly associated with any of the psychometric test results (data not shown), nor modified the association between these results and the treatment group (data not shown).

\section{Discussion}

The results of the present study show that a slight rise in TV and a reduction in thyroid function (increased TSH and decreased FT3 and FT4) were produced during pregnancy in women with insufficient iodine intake (median urinary iodine $<150 \mu \mathrm{g} / 1)^{(9)}$. The physiological decrease in thyroid function following the first trimester in iodine-sufficient women was largely mediated by decreased TSH receptor

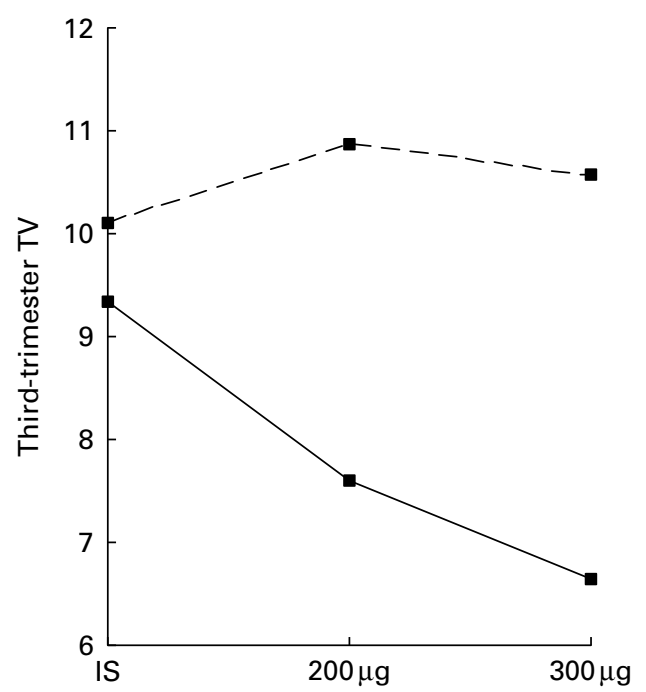

Fig. 2. Thyroid volume (TV) $\left(\mathrm{cm}^{3}\right)$ in the third trimester in the three groups of pregnant women (iodised salt (IS), 200 and $300 \mu \mathrm{g} /$ potassium iodide/d) according to whether the women had been taking iodised salt before pregnancy for more than 1 year $(-)$ or not $(--)(P=0.01$, adjusted for the intervention group). 

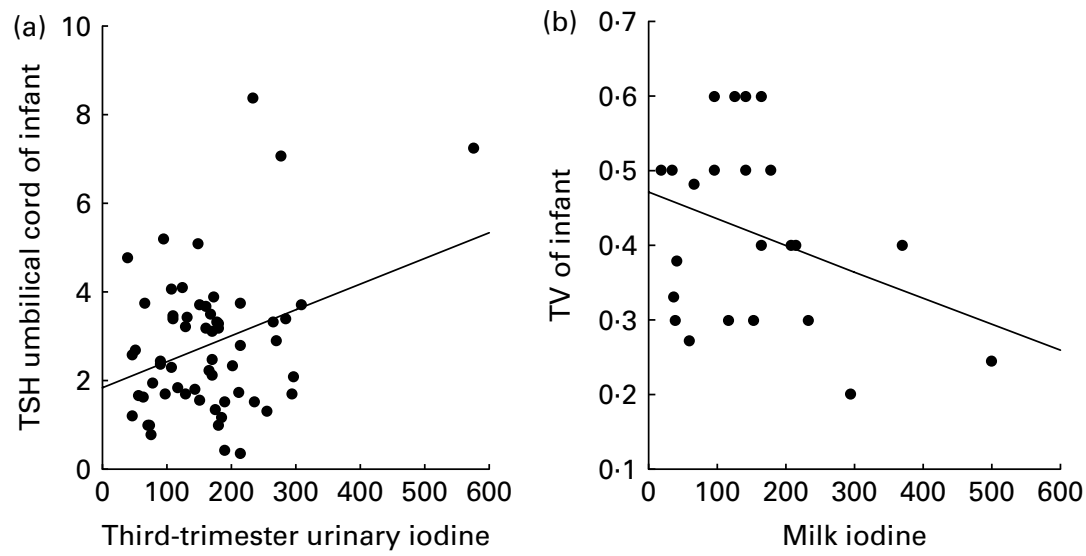

Fig. 3. (a) Correlation between cord blood thyroid-stimulating hormone (TSH) levels $(\mu \mathrm{lU} / \mathrm{ml})$ and urinary iodine in the third trimester ( $\mu \mathrm{g} / \mathrm{l})(r 0.40, P<0.0001$, adjusted for gestational age, third trimester TSH and treatment group). (b) Correlation between newborn thyroid volume (TV) (cm ${ }^{3}$ ) and iodine concentration in breast milk $(\mu \mathrm{g} / \mathrm{l})(r-0.43, P=0.04$, adjusted for gestational age $)$.

stimulation as serum human chorionic gonadotropin levels fall. Correlation existed neither between the two changes, nor with iodine intake, though the changes in TV seem to depend on the time the mother has been exposed to iodine consumption. The results also show that the neurocognitive capacity of the study children was not significantly associated with the form of iodine administration, either as salt fortified with iodine or as KI supplements.

Pregnancy is a period of intense changes in thyroid function in order to face the increased need for thyroid hormones, both maternal and fetal. Nutritional deficiency of iodine during the first half of pregnancy is associated with possible damage in the brain development of the offspring ${ }^{(18)}$. Clinically, iodine deficiency in schoolchildren has been associated with a reduction in auditory threshold ${ }^{(19)}$ and lower scores on the psychomotor and mental development tests ${ }^{(20)}$. Also, intellectual development of children at 25-30 months of age is associated with abnormalities of maternal thyroid at 16-20 weeks of gestation. Maternal subclinical hypothyroidism, hypothyroxinaemia or euthyroidism with elevated thyroid peroxidase antibody titres were all statistically significant predictors of lower motor and intellectual development at 25-30 months ${ }^{\text {(21). }}$. However, the iodine status of women in this study was not measured, because China is mostly iodine sufficient, thus the women in this study were unlikely to be iodine deficient.

In 2007, the WHO fixed the recommended daily intake of iodine during pregnancy at $250 \mu \mathrm{g}^{(9)}$. An adequate iodine intake can be achieved by increasing the consumption of iodine-rich food, replacing common salt with IS or prescribing pharmacological supplements of $\mathrm{KI}^{(17,22,23)}$. This increase should be maintained over time and occasionally submitted to periodic epidemiological vigilance, which is not always the case. Indicators of iodine deficiency have also been found in the USA among vulnerable groups, such as pregnant women $^{(24)}$.

Although the benefit of iodine supplementation in severe iodine deficiency is well established ${ }^{(8,12)}$, the benefits of iodine supplementation for mild-to-moderate iodine-deficient pregnant women are unclear ${ }^{(2,8,10)}$. Several studies have evaluated the impact of the administration of pharmacological supplements of KI during pregnancy. The effect of these supplements on the neurocognitive development of children is still under discussion ${ }^{(2,4,10)}$. A prior study by our group found that the use of pharmacological supplements of $300 \mu \mathrm{g} / \mathrm{d}$ of KI not only has no prejudicial effects on infant brain development but might also be beneficial in mothers with insufficient iodine intake ${ }^{(2)}$. The present study was

Table 2. Bayley test results in children according to treatment group (Mean values and standard deviations)

\begin{tabular}{|c|c|c|c|c|c|c|c|c|}
\hline & \multicolumn{2}{|c|}{ lodised salt } & \multicolumn{2}{|c|}{$200 \mu \mathrm{g} \mathrm{KI}$} & \multicolumn{2}{|c|}{$300 \mu \mathrm{g} \mathrm{KI}$} & \multirow[b]{2}{*}{ Crude $P^{\star}$} & \multirow[b]{2}{*}{$P^{\star \star}$} \\
\hline & Mean & SD & Mean & SD & Mean & SD & & \\
\hline$n$ & \multicolumn{2}{|c|}{25} & \multicolumn{2}{|c|}{47} & \multicolumn{2}{|c|}{30} & & \\
\hline MDS & 111.6 & 19.5 & $98 \cdot 2$ & 19.8 & $116 \cdot 2$ & $23 \cdot 2$ & 0.001 & 0.12 \\
\hline PDS & 48.4 & 8.7 & 42.0 & 9.6 & $48 \cdot 6$ & 6.7 & 0.001 & 0.36 \\
\hline MDI & $105 \cdot 6$ & $10 \cdot 6$ & 101.3 & 14.0 & 104.5 & 11.9 & 0.33 & 0.16 \\
\hline PDI & 100.9 & 11.5 & 94.2 & 13.6 & $98 \cdot 6$ & $16 \cdot 7$ & 0.13 & 0.10 \\
\hline TDI & 206.9 & $18 \cdot 2$ & 195.5 & $25 \cdot 3$ & 203.5 & 25.5 & 0.12 & 0.06 \\
\hline
\end{tabular}

MDS, Mental Development Scale; PDS, Psychomotor Development Scale; MDI, Mental Development Index; PDI, Psychomotor Development Index; TDI, Total Development Index. *ANOVA models.

${ }^{\star \star} P$ values adjusted for gestational age at birth, infant age and urinary iodine in the third trimester. 
carried out using very similar methods to our earlier study. Unlike the earlier study ${ }^{(2)}$, however, the present results showed no significant differences between the intervention groups in the neurocognitive development of children, as measured with the Bayley test. However, the control group in the earlier study did not consume IS and had greater iodine deficiency $(87 \cdot 6$ (SD 62.0) $\mu \mathrm{g} / \mathrm{l})$, whereas in the present study, the control group (pregnant women with less iodine deficiency, $145 \cdot 1$ (SD 89.7) $\mu \mathrm{g} / \mathrm{l}$ ) did take iodine in the form of IS. Second, the prior study was not a randomised controlled trial, and thus it is very different from the present study. In the earlier study, women who were supplemented with iodine may have been different from women who were in the control group. Also, children of the control and $300 \mu \mathrm{g}$ groups were not assessed at the same age, a major limitation, unlike the present study, where the age of the children in the three groups was similar. This may be the explanation for the differences found between the two studies.

The present study has a few limitations. Some of the women may not have strictly fulfilled the recommendations about consuming IS or about the prescription of pharmacological supplements of KI. Even so, the results concerning urinary iodine levels in each group are coherent with what was to be expected. It is also possible that the sample size in some stratifications was not sufficient to guarantee the absence of type II error. Furthermore, the age of the children ranged between 6 and 18 months. However, the MDI, PDI and TDI are comparable, independently of the age level at which they are obtained ${ }^{(25)}$, as is also shown by the lack of correlation between the indices and the age of children. The present study also has some strengths. The women received pharmacological supplements with KI, regardless of folic acid supplementation, but not in association with iodineenriched multivitamin supplements. Previous studies have shown that the use of iodine-enriched multivitamins is not clearly associated with the expected urinary iodine levels ${ }^{(26,27)}$. Those women who took IS before becoming pregnant were also identified.

Certain explanations exist for the differences with the previous study, in which the children of those women who took $300 \mu \mathrm{g} \mathrm{KI} / \mathrm{d}$ during pregnancy had better results on psychometric tests between the first and second years of life ${ }^{(2)}$. In Spain the intake of iodine has risen considerably since the first study was performed ${ }^{(28)}$. This increase is probably more related to the increase in iodine in milk than to an increased consumption of $\mathrm{IS}^{(16)}$. Indeed, whereas at the start of the earlier study the women had a mean urinary iodine level of $80 \mu \mathrm{g} / \mathrm{l}^{(2)}$, the median at the start of the present study was $109 \mu \mathrm{g} / \mathrm{l}$. Taken together, these results suggest that the effect of a KI supplement on the neurocognitive development of children depends on prior nutritional status.

Although it was not one of the main objectives of the present study, information about the time that the women were taking IS before becoming pregnant was included at the start of the study. In the present study, women who had consumed IS for at least 1 year before becoming pregnant had higher urinary iodine levels not only in the first trimester, as expected, but also in the second and third trimesters, irrespective of the group. In addition, TV in these women was less in the third trimester, suggesting the presence of a satisfactory iodine reserve and more stable thyroid iodine metabolism, as opposed to those women who started consuming IS only after learning that they were pregnant. Moleti et $a{ }^{(29)}$ showed that the prolonged use of IS significantly improved maternal thyroid function and reduced the risk of maternal thyroid deficiency during pregnancy, probably due to the normalisation of intra-thyroid iodine deposits.

Some of the results in pregnant women with insufficient iodine intake concerning iodine consumption found in the present study had already appeared in our previous report ${ }^{(2)}$, for example: the progressive rise in maternal TSH whatever the consumption of iodine, especially in the third trimester of the pregnancy ${ }^{(26,29)}$; or the correlation between third trimester urinary iodine levels and cord blood TSH. Both these findings again highlight the paradoxical correlations found between iodine intake and thyroid function, particularly with maternal and infant TSH, even in populations with sufficient intake of iodine.

Iodine requirements during pregnancy are known to rise $^{(8,13)}$, and guaranteeing adequate iodine intake by means of IS or supplements would seem a reasonable option ${ }^{(1,30)}$. However, the results of iodine intervention studies concerning thyroid function during pregnancy are far from conclusive. All the studies achieve an increase in urinary iodine levels, but there is no clear dose-response relationship with urinary iodine levels themselves or with the levels of TSH, FT4, FT3 or thyroglobulin ${ }^{(8)}$. The reduction in maternal thyroid function seen during pregnancy may be partly due to relative iodine deficiency resulting from the negative balance in iodine reserves in the body of a pregnant woman ${ }^{(8)}$. In the present study, though, the reduction in maternal thyroid function was to a certain extent independent of the amount of iodine taken during pregnancy.

The results show that urinary iodine levels in the third trimester were higher in women who received KI and that a positive correlation existed between the third-trimester urinary iodine levels and the cord blood TSH values. We had already found these results in a previous study in a population with a lower intake of iodine ${ }^{(2)}$. Cord blood TSH values between 7 and $9 \mathrm{mIU} / \mathrm{ml}$ in women with moderate iodine deficiency who had received a daily KI dose of $150 \mu \mathrm{g}$ were interpreted as evidence of an undesirable and potentially harmful effect of iodine supplementation ${ }^{(31)}$. However, more recent studies showed that, as opposed to what is expected, cord blood levels of TSH and FT4 correlated positively ${ }^{(18,32)}$. From this perspective, therefore, mildly elevated cord blood TSH values in women who had received iodine supplements should not be interpreted as potentially harmful but rather as a neonatal mechanism to guarantee adequate fetal FT4 levels.

In summary, in a population with insufficient iodine intake, the changes in maternal thyroid function during pregnancy were not associated with the form of iodine intake or with the dose given in the present study, but they were associated with the intake of IS before becoming pregnant. The neurocognitive development of infants was not associated with maternal iodine intake. The positive correlation found 
between urinary iodine levels in the third trimester and cord blood TSH levels merits further investigation. The results demonstrate the need to continue studying the physiological behaviour of maternal and neonatal thyroid function under different conditions of dietary iodine intake. Given that many countries lack epidemiological vigilance to guarantee a sufficient iodine intake, the question of whether systematic $\mathrm{KI}$ supplementation is recommended remains open.

\section{Acknowledgements}

CIBER de Diabetes y Enfermedades Metabólicas asociadas and CIBER de Fisiopatología de la Obesidad y Nutrición are ISCIII initiatives. The present study was undertaken with the aid of a grant from the Consejería de Salud of the Junta de Andalucía (PI-0469/2009/Consejería de Salud). The authors' contributions are as follows: P. S., I. V. and F. S. participated in the design of the study. P. S., F. S. and E. G.-F. performed the statistical analysis. C. G.-R., E. G.-F. and B. S. analysed the plasma and urine samples. P. S., M. B. and A. M. participated in the selection of the patients. J. M. measured TV by real-time ultrasound. J. A. M., A. R. and M. C. performed the evaluation of children's neurological development. All authors contributed to the writing of the manuscript, and read and approved the final manuscript. The authors declare that there is no conflict of interest.

\section{References}

1. Skeaff SA (2011) Iodine deficiency in pregnancy: the effect on neurodevelopment in the child. Nutrients 3, 265-273.

2. Velasco I, Carreira M, Santiago P, et al. (2009) Effect of iodine prophylaxis during pregnancy on neurocognitive development of children during the first two years of life. J Clin Endocrinol Metab 94, 3234-3241.

3. Ghassabian A, Bongers-Schokking JJ, Henrichs J, et al. (2011) Maternal thyroid function during pregnancy and behavioral problems in the offspring: the generation $\mathrm{R}$ study. Pediatr Res 69, 454-459.

4. Berbel P, Mestre JL, Santamaría A, et al. (2009) Delayed neurobehavioral development in children born to pregnant women with mild hypothyroxinemia during the first month of gestation: the importance of early iodine supplementation. Thyroid 19, 511-519.

5. Craig WY, Allan WC, Kloza EM, et al. (2012) Mid-gestational maternal free thyroxine concentration and offspring neurocognitive development at age two years. J Clin Endocrinol Metab 97, 22-28.

6. Leung AM, Pearce EN \& Braverman LE (2011) Iodine nutrition in pregnancy and lactation. Endocrinol Metab Clin North Am 40, 765-777.

7. Expert Group on Vitamins and Minerals (2003) Safe Upper Levels for Vitamins and Minerals. London: Food Standards Agency.

8. Zimmermann MB (2007) The adverse effects of mild-to-moderate iodine deficiency during pregnancy and childhood: a review. Thyroid 17, 829-835 (erratum in: Thyroid 2008 18, 97).

9. WHO Secretariat, Andersson M, de Benoist B, et al. (2007) Prevention and control of iodine deficiency in pregnant and lactating women and in children less than 2-years-old: conclusions and recommendations of the Technical Consultation. Public Health Nutr 10, 1606-1611.

10. Rebagliato M, Murcia M, Espada M, et al. (2010) Iodine intake and maternal thyroid function during pregnancy. Epidemiology 21, 62-69.

11. Sang Z, Wei W, Zhao N, et al. (2012) Thyroid dysfunction during late gestation is associated with excessive iodine intake in pregnant women. J Clin Endocrinol Metab 97, E1363-E1369.

12. Pharoah PO \& Connolly KJ (1987) A controlled trial of iodinated oil for the prevention of endemic cretinism: a longterm follow-up. Int J Epidemiol 16, 68-73.

13. Domínguez I, Reviriego S, Rojo-Martínez G, et al. (2004) Iodine deficiency and thyroid function in healthy pregnant women. Med Clin (Barc) 122, 449-453.

14. Santiago P, Berrio M, Olmedo P, et al. (2011) [Reference values for thyroid hormones in the population of pregnant women in Jaen (Spain)]. Endocrinol Nutr 58, 62-67.

15. Benotti J \& Benotti N (1963) Protein bound iodine, total iodine and protein and butanol extractable iodine by partial automation. Clin Chem 9, 408-416

16. Soriguer F, Gutierrez-Repiso C, Gonzalez-Romero S, et al. (2011) Iodine concentration in cow's milk and its relation with urinary iodine concentrations in the population. Clin Nutr 30, 44-48.

17. Soriguer F, Gutiérrez-Repiso C, Rubio-Martin E, et al. (2011) Iodine intakes of $100-300 \mu \mathrm{g} / \mathrm{d}$ do not modify thyroid function and have modest anti-inflammatory effects. Br J Nutr $\mathbf{2 5}$, $1-8$.

18. De Escobar GM, Obregón MJ \& del Rey FE (2007) Iodine deficiency and brain development in the first half of pregnancy. Public Health Nutr 10, 1554-1570.

19. Soriguer F, Millón MC, Muñoz R, et al. (2000) The auditory threshold in a school-age population is related to iodine intake and thyroid function. Thyroid 10, 991-999.

20. Santiago-Fernandez P, Torres-Barahona R, Muela-Martínez JA, et al. (2004) Intelligence quotient and iodine intake: a cross-sectional study in children. J Clin Endocrinol Metab 89, 3851-3857.

21. Li Y, Shan Z, Teng W, et al. (2010) Abnormalities of maternal thyroid function during pregnancy affect neuropsychological development of their children at 25-30 months. Clin Endocrinol (Oxf) 72, 825-829.

22. Untoro J, Mangasaryan N, de Benoist B, et al. (2007) Reaching optimal iodine nutrition in pregnant and lactating women and young children: programmatic recommendations. Public Health Nutr 10, 1527-1529.

23. Charlton K \& Skeaff S (2011) Iodine fortification: why, when, what, how, and who? Curr Opin Clin Nutr Metab Care 14, 618-624.

24. Caldwell KL, Makhmudov A, Ely E, et al. (2011) Iodine status of the U.S. population. National Health and Nutrition Examination Survey, 2005-2006 and 2007-2008. Thyroid 21, 419-427.

25. Alonso Tapia J (1992) Evaluación del conocimiento, la inteligencia y las aptitudes. Aportaciones de la psicología cognitiva. In Introducción a la evaluación psicológica I, pp. 349-383 [R Fernández-Ballesteros, editor]. Madrid: Pirámide.

26. Murcia M, Rebagliato M, Iñiguez C, et al. (2011) Effect of iodine supplementation during pregnancy on infant neurodevelopment at 1 year of age. Am J Epidemiol 173, 804-812.

27. García-Fuentes E, Gallo M, García L, et al. (2008) Amniotic fluid iodine concentrations do not vary in pregnant women with varying iodine intake. Br J Nutr 99, 1178-1181. 
28. Vila L, Serra-Prat M, de Castro A, et al. (2011) Iodine nutritional status in pregnant women of two historically different iodine-deficient areas of Catalonia, Spain. Nutrition 27, $1029-1033$

29. Moleti M, Di Bella B, Giorgianni G, et al. (2011) Maternal thyroid function in different conditions of iodine nutrition in pregnant women exposed to mild-moderate iodine deficiency: an observational study. Clin Endocrinol (Oxf) 74, $762-768$
30. Yarrington CD \& Pearce EN (2011) Dietary iodine in pregnancy and postpartum. Clin Obstet Gynecol 54, 459-470.

31. Nøhr SB \& Laurberg P (2000) Opposite variations in maternal and neonatal thyroid function induced by iodine supplementation during pregnancy. J Clin Endocrinol Metab 85, 623-627.

32. Hume R, Simpson J, Delahunty C, et al. (2004) Human fetal and cord serum thyroid hormones: developmental trends and interrelationships. J Clin Endocrinol Metab 89, 4097-4103. 